\title{
Desarrollo de las habilidades motoras fundamentales en función del sexo y del índice de masa corporal en escolares
}

\author{
Development of the fundamental motor skills depending \\ on the sex and the body mass index in schoolboys
}

\section{Desenvolvimento das habilidades motoras fundamentais em função do sexo e do índice de massa corporal de escolares}

\author{
Luciano Bucco-dos Santos y Marta Zubiaur-González
}

Universidad de León, España

\begin{abstract}
RESUMEN: El objetivo de este estudio fue analizar y determinar objetivamente si existen diferencias en los parámetros del Desarrollo Motor (DM) en cuanto al género y al Índice de Masa Corporal (IMC) de niños con sobrepeso/obesidad y niños normopeso. Se midieron las características antropométricas y se evaluó el DM con la Escala de Desarrollo Motor EDM a 284 niños sanos de 6 a 10 ańos de edad de la Comunidad de Cruz Alta Provincia Rio Grande do Sul, Brasil. Se observaron diferencias en el DM relacionadas con el género y con el IMC (sobrepeso/obesidad versus normopeso). Los niños y niñas con normopeso tienen un DM significativamente superior que los niños con sobrepeso/obesidad $(\mathrm{p} \leq 0,02)$. Los niños están significativamente $(\mathrm{p} \leq 0,01)$ más desarrollados motrizmente que las niñas en las estructuras motoras Equilibrio ( $E)(p \leq 0,03)$, Esquema Corporal (EC) $(\mathrm{p} \leq 0,01)$ y Organización Espacio-Temporal (OET) $(\mathrm{p} \leq 001)$. Las niñas son significativamente superiores ( $\mathrm{p} \leq 0,01)$ solo en las estructuras Motricidad Fina $(M F)(p \leq 0,00)$ y Motricidad Gruesa $(M G)(p \leq 0,01)$.

Palabras clave: desarrollo motor, obesidad, sobrepeso, escolares

ABSTRACT: The target of this study was to analyze objectively and to determine if differences exist in the parameters of the Motor Development to the sex and body mass index of children with over weight / obesity and children normal weight. The characteristics measured themselves anthropometrics and they will evaluate the (Motor Development with the Scale of Motor Development to 284 healthy children from 6 to 10 years of age of the Community of Cruz Alta state Rio do Sul, Brazil. The Motor Development observed differences in to the gender and with the body mass index (over weight / obesity versus normal weight). The boys and girls with
\end{abstract}

normal weight have more motor development that the children with over weight / obesity significantly ( $\mathrm{p} \leq 0,02)$. The boys are significantly $(\mathrm{p} \leq 0,01)$ more motor developed that the girls in the motor structures as Balance (E) $(\mathrm{p} \leq 0,03)$, Corporal Scheme (EC) $(\mathrm{p} \leq 0,01)$ and Organization I spread Storm $(\mathrm{OET})(\mathrm{p} \leq 001)$. The girls are significantly more developed in Fine Motor Skill ( $\mathrm{p} \leq 0,00)$ and Gross Motor Skill ( $\mathrm{p} \leq 0,01)$.

Key words: motor development, obesity, overweight, schoolboys

Resumo: O Objetivo desse estudo foi de analisar e determinar objetivamente se existem diferenças nos parâmetros do Desenvolvimento Motor (DM) referente ao gênero e o Índice de Massa Corporal (IMC) de crianças com sobrepeso/ obesidade e crianças normo-peso. Foram realizadas mediçóes antropométricas e avaliado o DM com a Escala de Desenvolvimento Motor (EDM) em 284 crianças saudáveis de 6 a 10 anos de idade da Comunidade de Cruz Alta, Estado do Rio Grande do Sul, Brasil. Observaram-se diferenças no DM relacionadas com o gênero e com o IMC (sobrepeso/ obesidade versus normo-peso). As crianças com normo-peso tem um DM significativamente superior que as crianças com sobrepeso/ obesidade $(\mathrm{p} \leq 0,02)$. Os meninos estáo significativamente mais $(\mathrm{p} \leq 0,01)$ desenvolvidos motoramente que as meninas nas estruturas motoras de Equilíbrio $(\mathrm{p} \leq 0,03)$, Esquema Corporal (EC) $(\mathrm{p} \leq 0,01)$ e Organização Espaço-Temporal (OET) $(\mathrm{p} \leq 001)$. As meninas são significativamente superiores $(\mathrm{p} \leq 0,01)$ somente nas estruturas Motricidade Fina (MF) ) ( $\mathrm{p} \leq 0,00)$ e Motricidade Grossa (MG) ( $\mathrm{p} \leq$ 0,01).

Palavras Chave: desenvolvimento motor, obesidade, sobrepeso, escolares.

\section{Introducción}

En el transcurrir de la vida se producen cambios de índole físico, motor, cognitivo, social y emocional originados por las limitaciones individuales, las experiencias vividas, por restricciones del contexto, por la especificidad y complejidad de las actividades que se presentan a lo largo de la evolución del

Dirección para correspondencia: Universidad de León. Facultad de Ciencias de la Actividad Física y del Deporte Campus de Vegazana S/N, 24071 León. España

mzubg@unileon.es niño de forma que las diferentes condiciones pueden animar o desanimar al niño a explorar el movimiento (Gallahue y Ozmun, 2006; Haywood y Getchell, 2004).

El desarrollo de las habilidades motoras fundamentales, desde la perspectiva de una práctica adecuada al nivel de desarrollo infantil, debería plantearse como una prioridad en los programas de movimiento (Ruiz, 2004). Estas habilidades motoras se consideran bloques de construcción indispensables tanto para el desarrollo de actividades de movimiento como para la especialización de habilidades motoras espe- 
cíficas (Haywood y Getchell, 2004). Es importante resaltar que estos patrones fundamentales no emergen naturalmente durante la infancia, son el resultado de varios factores que, en constante interacción, influencian el desarrollo motor del niño, como el contexto de enseñanza, la motivación, el desarrollo neurológico, las condiciones sociales y culturales y las experiencias pasadas (Haywood y Getchell, 2004).

El niño está en constante evolución, y a medida que su edad avanza sus cambios funcionales mejoran a causa de las experiencias vividas, (Massa y Ré, 2010). Estas modificaciones constantemente se alteran debido la interacción con el ambiente y con la tarea propuesta (Haywood y Getchell, 2004). El movimiento del niño se desarrolla y mejora con esa interacción (Massa y Ré, 2010), produciéndose modificaciones de orden cuantitativo denominadas de crecimiento físico, como el aumento de estatura y peso corporal, y de orden cualitativo, como la adquisición y mejora de las funciones motoras (Malina y Bouchard, 2002, Gallahue y Ozmun, 2006, Ruiz et al., 2007).

Los escolares que presentan una baja percepción de competencia motriz no se ven capaces de realizar muchas de las tareas que sus iguales realizan sin grandes dificultades (Ruiz et al., 2007), asimismo, manifiestan una menor motivación hacia la práctica de actividades físicas y deportivas, lo que puede llevarles a tener dificultades de relación con sus compañeros, provocando episodios de soledad, aislamiento, rechazo y ridículo en el patio de recreo o en momentos de juego. Actualmente se ha observado que el ser humano, en especial los niños y adolescentes, vienen practicando menos actividad física convirtiéndose esta inactividad en una de las grandes causas del aumento del peso corporal (Martínez-López et al., 2009), y de la baja competencia motriz en los niños (Ruiz et al., 2007). La obesidad, directamente correlacionada con la inactividad física, se considera hoy día como una enfermedad crónica, compleja y multifactorial, que suele iniciarse en la infancia y la adolescencia provocando el riesgo de padecer numerosos problemas de salud (García-Martos et al., 2010). Las recomendaciones de actividad física para la salud actualmente vigentes exponen que todos los niños/as y adolescentes deberían participar en actividad de intensidad entre moderada y vigorosa, durante al menos una hora al día, y al menos dos veces a la semana (Ekelund et al., 2004). Sin embargo, esta actividad debería ayudar a mejorar y/o mantener la fuerza muscular, la flexibilidad y la salud ósea. El desempeño motor se mejora con la práctica pero la ejecución motora de un niño está correlacionada con la cantidad y diversidad de propuestas motrices que se le ofrecen.

Por otro lado, numerosos estudios ponen de manifiesto que las niñas practican menos actividad física que los niños durante la preadolescencia y la adolescencia lo que puede repercutir en su desarrollo motor (luengo, 2007; Montil et al., 2005, Sallis et al, 2000)
Por todo lo expuesto, nuestro objeto de estudio consiste en evaluar y comparar la evolución de las características motoras de niños en función del género y del índice de masa corporal.

\section{Material y métodos}

\section{Participantes}

La población del presente estudio fue compuesta por niños de 6 a 10 años de la enseñanza fundamental de ambos géneros matriculados en la Red Pública Provincial de enseñanza de la ciudad de Cruz Alta, Provincia Río Grande del Sur, país Brasil, la cual está constituida por dieciocho escuelas con un total de 4.978 niños de 6 a 10 ańos. Fueron seleccionadas ocho escuelas públicas de las dieciocho, con un total de 2679 $(53,8 \%)$ niños de 6 a 10 años. Se seleccionaron tres escuelas de la región central y cinco de la región periférica de la ciudad, por presentar un mayor número de escolares matriculados. De las ochos escuelas pertenecientes al estudio, fueron seleccionados 324 nińos (12,9\%) con características corporales para el estudio. De los 324 niños seleccionados, 284 $(87,65 \%)$ fueron autorizados por sus padres para formar parte del estudio, los cuales presentaron un documento firmado manifestando su consentimiento para que sus hijos realizasen los tests, de tal forma que $141(49,64 \%)$ fueron niñas siendo clasificadas 110 normopeso y 31 sobrepeso/obesidad, y 143 $(50,35 \%)$ fueron niños de los cuales 98 normopeso y 45 sobrepeso/obesidad. La participación de los 284 niños por edad fue la siguiente: media general de la muestra $(M=8.13$ edad, $\mathrm{DT} \pm 1.23) 54$ de 6 ańos (19,01\%), 47 de 7 años (16,54\%), 53 de 8 años (18,66\%), 67 de 9 años (23,59\%), y, por último, 63 de 10 años (22,20\%), (tabla 2).

\section{Medidas Instrumentos}

Medición de características antropométricas. Para la clasificación y distribución de los 284 niños en los grupos se utilizó el patrón del Sistema de Evaluación del Estado Nutricional Infantil según el NCHS (National Center for Health Statistics, 2005) y CDC (Centers for Disease Control and Prevention, 2005) donde sus valores fueron obtenidos a través del IMC calculado a través de la formula: IMC = masa corporal (en $\mathrm{kg}$ )/estatura ${ }^{2}$ (en metros). El NCHS trabaja con percentiles a través del IMC desde las edades de 2 a 20 años de la siguiente forma: valores de percentil entre 5 y 85 corresponden a nińos con peso normal, valores entre 85 y 95 son niños con parámetros de sobrepeso y valores por encima de 95 son niños con parámetros corporales indicativos de obesidad. Los datos recolectados para evaluar el estado nutricional de los niños se basaron en el resultado del peso siendo utilizada una báscula electrónica portátil marca Plena, modelo MEA08128 con capacidad para $180 \mathrm{~kg}$, y para la talla el aparato es- 
tadiómetro marca Cardiomed, modelo WCS para hasta 212 $\mathrm{cm}$. de talla. Para el diagnóstico de la obesidad, sobrepeso y normopeso, se halló el IMC, teniendo como referencia los datos del NCHS, (2005).

Medición DM. Para evaluar el desempeño motor (DM), se ha utilizado el protocolo de pruebas EDM, Escala de Desarrollo Motor, este instrumento fue desarrollado por Francisco Rosa Neto, en 1996, en la Universidad de Zaragoza a partir de otros tests motores con el respaldo de autores clásicos como: Ozeretski, Brunet y Lezine, Berges y Lezine, Mira Stambak, Galifred- Granjon, Zazzo, Piaget y Head, Picp y Vayer. Fue creado para identificar y diagnosticar niños con dificultades de movimiento y coordinación, entre 2 y 11 ańos, formado por un conjunto de 150 tareas motrices que son aplicadas de acuerdo con la edad cronológica de cada niño; el test mide la evolución motora del control global del cuerpo. La fiabilidad del instrumento se estableció mediante test-retest en un periodo de cuatro semanas en una muestra espańola de 180 niños entre 2 a 11 años en 1996 para identificar y diagnosticar niños con dificultades de movimiento y coordinación.
Desde 1996 la escala está siendo utilizada en trabajos de las siguientes aéreas: Educación Física, Fisioterapia, Pedagogía y Medicina, siendo utilizada en varios estudios (Fonseca et al., 2008; Rosa Neto et al., 2010). Los tests motores de la escala se distribuyen en siete áreas de evaluación: motricidad fina, motricidad gruesa, equilibrio, esquema corporal organización espacial, organización temporal. Como está previsto en el manual del test, se anotan las puntuaciones que el sujeto obtiene en cada una de las tareas (número de pasos, altura superada, número de saltos, número de veces que ejecuta un movimiento). El manual ofrece normas para los escolares en intervalos anuales (de 2 a 11 años), presentando en dos de las cuatro tareas (salto con una pierna y saltos laterales) puntuaciones iguales para chicos y chicas. Estas puntuaciones son transformadas en un cociente motor de cada una de las tareas, posteriormente en un cociente motor global (Media 100, Desviación Típica 15). Para esta labor se emplearon las tablas con datos normativos de la escala EDM que ofrece una clasificación motora en meses y que fueron transformadas en años para este estudio (tabla 1).

Tabla 1. Clasificación de la EDM de acuerdo con el cociente motor.

\begin{tabular}{|c|c|c|}
\hline Cociente motor meses EDM & Cociente motor años & Clasificación \\
\hline$\leq 70$ meses & 5 años y 6 meses & problemático \\
\hline $79-70$ meses & 6 años y 6 meses a 5 años y 6 meses & inferior \\
\hline $89-80$ meses & 7 ańos y 6 meses a 6 ańos y 6 meses & normal bajo \\
\hline 109- 90 meses & 9 ańos y 6 meses a 7 ańos y 6 meses & normal medio \\
\hline $119-110$ meses & 10 años y 6 meses a 9 años y 6 meses & normal alto \\
\hline $129-120$ meses & 11 años y 6 meses a 10 años y 6 meses & superior \\
\hline$\geq 130$ meses & 11 años y 6 meses & mucho superior \\
\hline
\end{tabular}

\section{Análisis de datos}

Para la formación de los grupos se utilizaron como referencia las curvas del NCHS - National Center for Health and Statistics (2005). Para la clasificación del desempeño de las estructuras motoras se utilizaron los respectivos resultados de acuerdo con la EDM (tabla 1). Los resultados fueran analizados por medio de la media y desviación típica de las edades motoras, cronológicas, peso, talla e IMC. Para la comparación entre grupos se llevo a cabo un análisis de varianza factorial (ANOVA) de dos factores: género y normopeso vs sobrepeso/obesidad. Se utilizo la prueba de t de student para examinar las diferencias de género y las diferencias según los grupos de IMC y EDM vs estructuras motoras, y normopeso vs sobrepeso/obesidad. El criterio estadístico de significación aplicado en este estudio fue de $\mathrm{p} \leq 0,05$. Fue utilizado el test de normalidad de Kolmogorov-Smirnov para la muestra.

\section{Resultados}


Tabla 2. Distribución de la muestra de acuerdo con la edad, género y en cuanto la clasificación: normopeso sobrepeso /obesidad

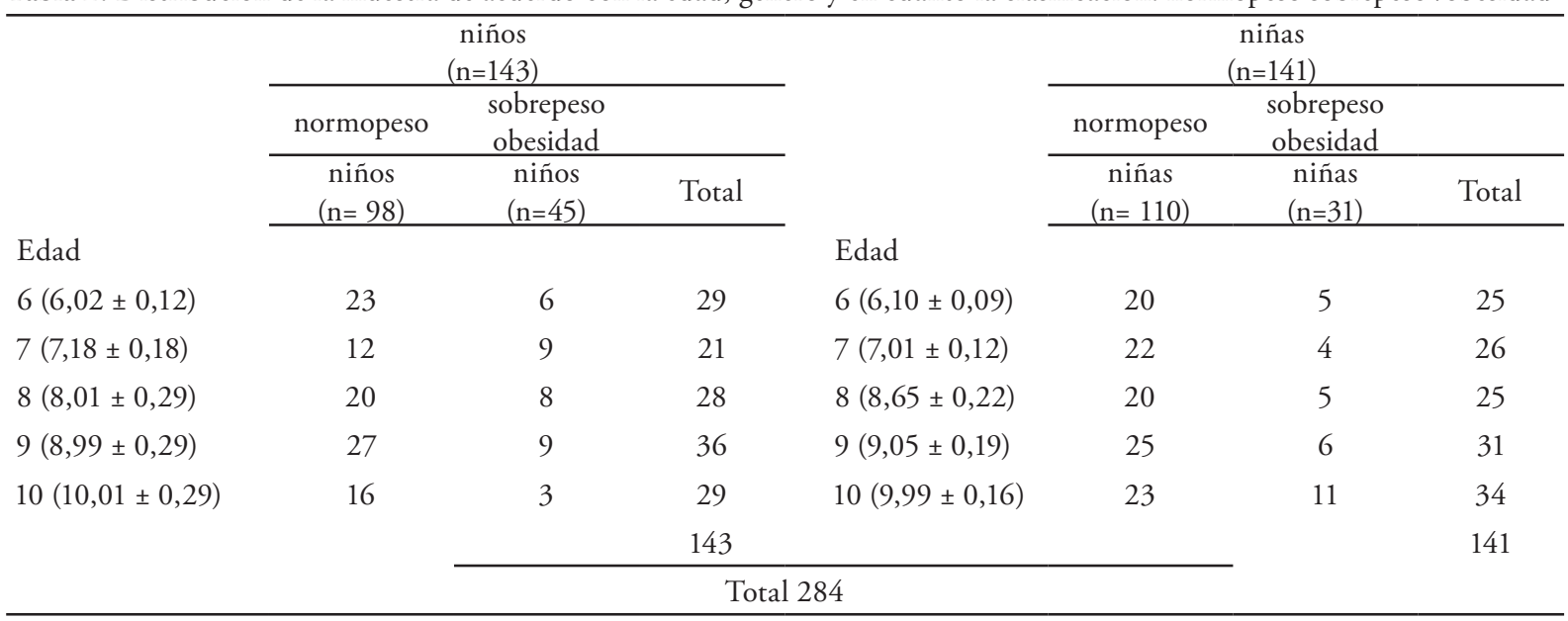

El efecto edad fue constatado para el peso talla e IMC respectivamente $(\mathrm{P} \leq 0,01),(\mathrm{P} \leq 0,03),(\mathrm{P} \leq 0,04)$ de forma que, tanto los niños normopeso como los sobrepeso/obesidad presentaron un aumento progresivo con el aumento de la edad. En la comparación entre grupos respecto al peso e IMC, fue detectado un efecto significativo respectivamente $(\mathrm{P} \leq 0,03)$, $(\mathrm{P} \leq 0,02)$, poniendo de manifiesto que los niños con parámetros de sobrepeso/obesidad presentaron valores superiores de los normopeso. Para la talla no se encontraron diferencias entre los grupos (tabla 3).

Tabla 3. Valores medios y (DT \pm ) del peso, talla e IMC de la muestra investigada de acuerdo con las edades y clasificación: normopeso sobrepeso/obesidad

\begin{tabular}{|c|c|c|c|c|c|c|}
\hline \multirow[b]{3}{*}{ Edad } & \multicolumn{2}{|c|}{ Peso } & \multicolumn{2}{|c|}{ Talla } & \multicolumn{2}{|c|}{ IMC $\left(\mathrm{kg} / \mathrm{m}^{2}\right)$} \\
\hline & $\begin{array}{c}\text { normopeso } \\
(\mathrm{n}=208)\end{array}$ & $\begin{array}{c}\text { sobrepeso } \\
\text { obesidad } \\
(n=76)\end{array}$ & $\begin{array}{l}\text { normopeso } \\
\quad(\mathrm{n}=208)\end{array}$ & $\begin{array}{c}\text { sobrepeso } \\
\text { obesidad } \\
(\mathrm{n}=76)\end{array}$ & $\begin{array}{l}\text { normopeso } \\
\quad(\mathrm{n}=208)\end{array}$ & $\begin{array}{c}\text { sobrepeso } \\
\text { obesidad } \\
(\mathrm{n}=76)\end{array}$ \\
\hline & niños & niños & niños & niños & niños & niños \\
\hline \pm 6 & $25,20 \pm 4,6$ & $29,33 \pm 7,2^{* \#}$ & $1,22 \pm 1,2^{*}$ & $1,18 \pm 2,3$ & $16,57 \pm 3,2$ & $18,82 \pm 2,2$ *\# \\
\hline \pm 7 & $28,30 \pm 5,6$ & $30,21 \pm 1,9^{* \#}$ & $1,28 \pm 2,3^{*}$ & $1,27 \pm 2,5$ & $17,32 \pm 2,6$ & $18,60 \pm 1,3^{* \#}$ \\
\hline \pm 8 & $34,23 \pm 2,2$ & $35,98 \pm 3,4^{* \#}$ & $1,35 \pm 1,6$ & $1,36 \pm 1,5^{*}$ & $18,02 \pm 2,2$ & $19,49 \pm 6,2 * \#$ \\
\hline \pm 9 & $38,52 \pm 2,7$ & $41,23 \pm 7,1$ *\# & $1,42 \pm 1,5$ & $1,45 \pm 2,4^{*}$ & $18,84 \pm 4,5$ & $20,62 \pm 9,3^{* \#}$ \\
\hline \pm 10 & $37,25 \pm 3,6$ & $39,12 \pm 3,2^{* \#}$ & $1,45 \pm 2,7^{*}$ & $1,42 \pm 3,3$ & $20,03 \pm 2,4$ & $23,15 \pm 3,9^{* \#}$ \\
\hline F-edade & \multicolumn{2}{|c|}{$412 *$} & \multicolumn{2}{|c|}{$0,89^{*}$} & \multicolumn{2}{|c|}{$410^{*}$} \\
\hline F-grupo & \multicolumn{2}{|c|}{$36,54^{\#}$} & \multicolumn{2}{|c|}{85,23} & \multicolumn{2}{|c|}{$18,52^{\#}$} \\
\hline
\end{tabular}

*Diferencias significativas entre edad versus: peso talla e IMC $\mathrm{p} \leq 0,05^{* \#}$ Diferencias significativas entre los grupos normopeso versos sobrepeso/obesidad, peso, talla e IMC $\mathrm{p} \leq 0,05^{\#}$

Se encontraron diferencias significativas en el DM en función del género y grupo (normopeso vs sobrepeso/obesidad). El ANOVA indicó una diferencia significativa en cuanto al género para los niños normopeso vs sobrepeso/obesidad. Cuando se dividió la muestra por géneros, se encontraron diferencias significativas en la EMG (edad motora general) $(p \leq 0,02)$ equilibrio $(E)(p \leq 0,01)$, esquema corporal (EC) $(p \leq$ $0,04)$ organización espacial $(\mathrm{OE})(\mathrm{p} \leq 0,02)$ y organización temporal $(\mathrm{OT})(\mathrm{p} \leq 0,03)$ a favor de los niños. Las niñas fue- ran superiores motoramente en las estructuras motricidad fina $(\mathrm{MF})(\mathrm{p} \leq 0,01)$ y gruesa $(\mathrm{MG})(\mathrm{p} \leq 0,03)($ Tabla 4$)$. En cuanto al efecto comparativo de las variables motoras y EDM se observaron efectos significativos entre niños y niñas en la estructura (OT) organización temporal ( $\mathrm{p} \leq 0,03)$, siendo las niñas inferiores. Para el efecto comparativo variables motoras e IMC no se encontraron diferencias significativas en esta muestra. 
Tabla 4. Perfil motor de las variables: comparación cuanto al género, EDM e IMC media y (DT \pm ).

\begin{tabular}{|c|c|c|c|c|c|}
\hline \multirow[b]{2}{*}{ Variables } & \multicolumn{2}{|c|}{ niños $(\mathrm{n}=143)$} & \multicolumn{2}{|c|}{ niñas $(\mathrm{n}=141)$} & \multirow[b]{2}{*}{$\mathrm{p}<0,05$} \\
\hline & Media años & $\mathrm{DT} \pm$ & Media años & $\mathrm{DT} \pm$ & \\
\hline EC- Edad Cronológica & 8,5 & 8,23 & 8,3 & 4,12 & 0,25 \\
\hline EMG- Edad Motora General & $8,3^{*}$ & 7,21 & 7,3 & 8,36 & 0,02 \\
\hline MF- Motricidad Fine & 8,5 & 9,36 & $9,2 *$ & 5,63 & 0,01 \\
\hline MG-Motricidad Gruesa & 8,8 & 9,23 & $9,0 *$ & 7,56 & 0,03 \\
\hline E- Equilibrio & $9,2 *$ & 6,13 & 6,9 & 8,65 & 0,01 \\
\hline EC-Esquema Corporal & $8,8^{*}$ & 8,58 & 7,0 & 7,23 & 0,04 \\
\hline OE- Organización Espacial & $7,5^{*}$ & 9,03 & 7,1 & 8,55 & 0,02 \\
\hline OT- Organización Temporal & $6,8^{* \#}$ & 9,16 & $6,5^{\#}$ & 8,33 & 0,03 \\
\hline f- EDM & $45,32^{\#}$ & & $39,45^{\#}$ & & \\
\hline f- IMC & 13,45 & & 22,12 & & \\
\hline
\end{tabular}

Los niños normopeso vs sobrepeso/obesidad fueron significativamente mejores que sus compañeros en cuatro estructuras: equilibrio $(E)(p \leq 0,02)$, esquema corporal $(E C)(p \leq 0,03)$ organización espacial $(\mathrm{OE})(\mathrm{p} \leq 0,01)$ y organización temporal (OT) $(\mathrm{p} \leq 0,03)$. En cuanto al efecto comparativo del perfil motor de las variables y EDM entre el mismo grupo se encontraron diferencias significativas para los niños normopeso en la variable $(\mathrm{OT})$ organización temporal $(\mathrm{p} \leq 0,01)$, para los sobrepeso/obesidad en las variables (OE) organización espacial $(\mathrm{p} \leq 0,02)$ y $(\mathrm{OT})$ organización temporal $(\mathrm{p} \leq 0,04)$. La variable
(OT) organización temporal presento un perfil motor debajo de la media EDM para los dos grupos, de forma que no se puede afirmar que el factor sobrepeso/obesidad sea prejudicial para esta variable, pudiendo estar relacionada esta diferencia con otros factores como la falta de oportunidades motrices. También fueron detectadas diferencias significativas, para el efecto comparativo perfil de las variables motoras e IMC, para el $(\mathrm{EC})$ esquema corporal $(\mathrm{p} \leq 0,01)(\mathrm{OE})$ organización espacial $(p \leq 0,01)$ y $(O T)$ organización temporal $(p \leq 0,01)$ en el grupo sobrepeso/obesidad (Tabla 5).

Tabla 5. Comparación del perfil motor de las variables, media y (DT \pm ) de los niños normopeso vs sobrepeso/obesidad.

\begin{tabular}{|c|c|c|c|c|c|}
\hline \multicolumn{6}{|l|}{ niños $(n=143)$} \\
\hline \multirow[t]{2}{*}{ Variables } & \multicolumn{2}{|c|}{ normopeso $(\mathrm{n}=98)$} & \multicolumn{3}{|c|}{ sobrepeso obesidade $(\mathrm{n}=45)$} \\
\hline & Media & $\mathrm{DT} \pm$ & Media & $\mathrm{DT} \pm$ & $\mathrm{p}<0,05$ \\
\hline EC-Edad Cronológica & 8,5 & 6,23 & 8,5 & 6,23 & 0,23 \\
\hline EMG- Edad Motora General & $8,4^{*}$ & 6,54 & $7,1^{+}$ & 5,32 & 0,01 \\
\hline MF- Motricidad Fine & 8,5 & 4,98 & 7,1 & 4,22 & 0,12 \\
\hline MG- Motricidad Gruesa & 8,8 & 3,33 & 6,9 & 6,54 & 0,23 \\
\hline E- Equilibrio & $8,8^{*}$ & 3,22 & 6,6 & 3,23 & 0,02 \\
\hline EC- Esquema Corporal & $8,5^{*}$ & 5,33 & $6,8^{+}$ & 7,63 & 0,03 \\
\hline OE- Organización Espacial & $7,1 *$ & 5,58 & $6,6^{\#+}$ & 6,96 & 0,01 \\
\hline OT- Organización Temporal & $6,5^{* \#}$ & 6,55 & $6,2^{\#+}$ & 7,23 & 0,03 \\
\hline f-EDM & \multicolumn{2}{|c|}{$765^{\#} 235^{\#}$} & & & \\
\hline $\mathrm{f}-\mathrm{IMC}$ & \multicolumn{2}{|l|}{265} & \multicolumn{2}{|l|}{$312^{+}$} & \\
\hline
\end{tabular}

Las niñas normopeso fueron significativamente mejores que sus compañeras, sobrepeso/obesidad, en cinco estructuras respectivamente, $(\mathrm{MG})$ motricidad gruesa, $(\mathrm{p} \leq 0,01),(\mathrm{E})$ equilibrio $(\mathrm{p} \leq 0,02),(\mathrm{EC})$ esquema corporal $(\mathrm{p} \leq 0,01)(\mathrm{OE})$ organización espacial $(\mathrm{p} \leq 0,03)$ y $(\mathrm{OT})$ organización temporal $(p \leq 0,03)$. En cuanto al efecto comparativo perfil motor de las variables e IMC entre el mismo grupo, fueron encontradas diferencias significativas para el grupo sobrepeso/obesidad 
en las variables (EC) esquema corporal $(p \leq 0,02)$, (OE) organización espacial $(\mathrm{p} \leq 0,02)$ y $(\mathrm{OT})$ organización temporal $(p \leq 0,04)$. Con respecto al efecto comparativo EDM y perfil motor de las variables entre el mismo grupo, se encontraron diferencias significativas para las niñas con sobrepeso/obesidad, $(\mathrm{OE})$ organización espacial $(\mathrm{p} \leq 0,01)$ y $(\mathrm{OT})$ organización temporal $(\mathrm{p} \leq 0,01)$ (Tabla 6$)$.

Tabla 6. Comparación del perfil motor de las variables, media y (DT \pm ) de las niñas normopeso vs sobrepeso/obesidad.

\begin{tabular}{|c|c|c|c|c|c|}
\hline \multicolumn{6}{|l|}{ niñas $(\mathrm{n}=141)$} \\
\hline \multirow[b]{2}{*}{ Variables } & \multicolumn{2}{|c|}{ normopeso $(\mathrm{n}=110)$} & \multicolumn{3}{|c|}{ sobrepeso obesidad $(\mathrm{n}=31)$} \\
\hline & Media & $\mathrm{DT} \pm$ & Media & $\mathrm{DT} \pm$ & $\mathrm{p}<0,05$ \\
\hline EC-Edad Cronológica & 8,3 & 6,63 & 8,4 & 6,23 & 0,12 \\
\hline EMG- Edad Motora General & $8,0^{*}$ & 3,03 & $6,7^{+}$ & 4,33 & 0,01 \\
\hline MF- Motricidad Fine & 8,0 & 5,98 & 7,2 & 3,69 & 0,18 \\
\hline MG- Motricidad Gruesa & $8,5^{*}$ & 5,23 & 6,9 & 3,55 & 0,01 \\
\hline E- Equilibrio & $8,5^{*}$ & 6,22 & 7,0 & 5,12 & 0,02 \\
\hline EC- Esquema Corporal & $8,3^{*}$ & 3,53 & $6,7^{+}$ & 3,65 & 0,01 \\
\hline OE- Organización Espacial & $7,5^{*}$ & 6,08 & $6,5^{\#+}$ & 7,85 & 0,03 \\
\hline OT- Organización Temporal & $7,5 *$ & 5,05 & $6,4^{\#+}$ & 4,03 & 0,03 \\
\hline f- EDM & 623 & & $298 \#$ & & \\
\hline $\mathrm{f}-\mathrm{IMC}$ & 244 & & $387^{+}$ & & \\
\hline
\end{tabular}

\section{Discusión}

\section{Desempeño motor y género}

Diversos estudios sobre el desarrollo motor reconocen la existencia de períodos críticos y demuestran una fuerte relación entre la coordinación motora en la infancia y en los años de vida posteriores (Gallahue y Ozmun, 2006, Ruiz et al., 2007), o sea, parece que los primeros ańos de vida componen un período crítico tanto para la adquisición de las habilidades motoras como para la participación en la práctica de actividades físicas (Ahnert y Schneider, 2007; Barnett et al., 2009; Stodden et al, 2008) de forma que, aproximadamente a los diez años de edad, se espera que el niño tenga un amplio dominio de las habilidades motoras fundamentales.

Este estudio presenta las diferencias motoras entre niños y niñas de seis a diez años con sobrepeso/obesidad versus normopeso medidos de forma objetiva. Se ha podido evidenciar un nivel superior de equilibrio (E) obtenido por parte de los niños (tabla 4), así como se observa que las habilidades más refinadas son características de las niñas, como la motricidad fina (MF), y la motricidad gruesa (MG). En relación al equilibrio (E), Cury y Magalhães (2006) encontraron variaciones a lo largo de la infancia hasta ocho años. Shumway-Cook y Woollacott (2001) encontraron una superioridad de los niños en todas las pruebas de habilidades motoras, desde los siete años de edad. Sin embargo, en los resultados del presente es- tudio, de las seis habilidades motoras analizadas, las niñas fueron superiores en motricidad fina (MF) y motricidad gruesa (MG) (tabla 4).

Aznar et al. (2010) y Nilsson et al. (2009) destacan la superioridad de los niños en las habilidades motoras gracias a las prácticas deportivas, y a la superioridad física, siendo los niños más activos que las niñas. A las mismas conclusiones llegan Nieto et al. (2011) observando el comportamiento de niños y niñas: los niños exploran mucho más los espacios cuando se les proporcionan actividades lúdicas, pasando por casi todos los elementos; sin embargo las niñas dan preferencia a largos periodos de conversaciones en grupos de dos a tres compañeras. El estudio evidenció que los niños, participaban más en juegos colectivos organizados, como el fútbol, el baloncesto; las niñas se quedaban pasivas, muchas veces en la condición de espectadoras.

Todos estos resultados corroboran la hipótesis de que los niños son más activos que las niñas, y por ello tienden a tener un repertorio motriz más equilibrado y desarrollado (Sirard y Pate, 2001; Sallis et al., 2000). De acuerdo con Malina et al. (2004) las niñas cuando comienzan a interesarse por la práctica deportiva son, en general, dos años mayores que los niños, eso puede producir desventajas en el aprendizaje motor.

Entre los cinco y diez años de edad hay una gran evolución en la coordinación y control motor, facilitando el refinamiento de las habilidades motoras, cada vez mas complejas (Gallahue; Ozmun, 2006). De acuerdo con Malina et al. (2004) en este 
periodo, los niños son capaces de comprender las reglas del deporte y participar en programas estructurados siendo de gran importancia actividades que envuelven una amplia diversidad de movimientos. En esta fase se produce un aumento relativamente constante de la fuerza, velocidad y resistencia, especialmente cuando están presentes los estímulos ambientales adecuados (Malina et al., 2004). De ahí que sea tan importante el ofrecer los suficientes estímulos para la evolución de las capacidades del niño, preferentemente en situaciones que privilegien el desarrollo de la coordinación (Williams et al., 2008).

En lo referente al nivel de coordinación motora y género, se observó que los niños poseen niveles más elevados que las niñas. Estas diferencias, según Valdivia et al. (2008), se deben, muchas veces, a la diversidad de oportunidades en el medio escolar y familiar.

De acuerdo con Malina et al. (2004), Rogol et al. (2002) las diferencias en el desempeño motor entre niños y niñas son pequeñas, siempre que ambos tengan oportunidad de práctica motora. Pero todavía, en el caso de las niñas, por cuestiones culturales no tienen el mismo acceso al movimiento que los niños, hecho que puede perjudicar la adquisición de las habilidades motoras (Tucker, 2008).

Faustino et al. (2004) han investigado el efecto de la actividad física regular en niños de enseńanza básica, y constataron que, tanto en el pre-test como en el post-test, los niños obtienen mejores resultados que las niñas. La superioridad de los niños en relación a las niñas se verifica también en el estudio de Berleze et al. (2007); estas diferencias están relacionadas tanto con factores morfo-funcionales, como con factores socio-culturales (Malina et al., 2004). En este estudio se observaron diferencias a favor de los nińos en las estructuras motoras de equilibrio, esquema corporal, organización espacial y organización temporal. Especialmente es destacable la diferencia del equilibrio por ser una actividad refinada y que en teoría sería del dominio de las niñas, manifestándose los niños de nuestro estudio claramente superiores.

Las nińas se mostraran superiores en las variables motricidad fina $y$, curiosamente, motricidad gruesa, variable ésta donde se podría esperar una superioridad de los niños al priorizar actividades de lanzamiento de balón. La actividad de lanzamiento, de acuerdo con Gallahue y Ozmun, (2006) forman parte esencialmente de las actividades de los niños, tales variables intervinientes podrían explicar esas diferencias en las pruebas entre nińos y nińas encontradas en este estudio, pues no se da igual oportunidad a unos y otras para que desarrollen su repertorio motriz. Con respecto al desarrollo motor de niños y nińas fue detectado un retraso motor en la variable organización temporal, en relación con la EDM. Este retraso puede estar relacionado con la falta de estimulación motriz, esto sumado a la falta de interés que caracteriza a los niños del momento actual.

\section{Desempeño motor e IMC}

Los resultados del presente estudio encuentran soporte en Feder et al. (2005) y Ozdirenc et al. (2005) los cuales han mostrado la creciente preocupación por los niveles pobres de aptitud e inactividad física en niños. Una gran cantidad de estudios han demostrado que existe una asociación beneficiosa entre la actividad física y la prevención de la obesidad (Ekelund et al., 2004; García-Martos et al., 2010). Un fenómeno que ha despertado gran atención, principalmente en el contexto escolar, es el aumento considerable de los niveles de sobrepeso/obesidad y disminución de las actividades funcionales-motora. Ese aumento, muchas veces es causado por la inactividad física y por el sedentarismo (Ortega et al., 2008); puede estar directamente conectado a la falta de experiencias motoras y de participación en programas de ejercicio físico, afectando, en ese contexto, a los niveles de coordinación motora (Ruiz et al., 2007; Tucker, 2008), así como al aumento de la prevalencia del sobrepeso y obesidad. Pelegrini et al., (2003) observaron que los niños con dificultades motoras en clases de educación física no realizaban actividades con la misma disposición que sus compañeros.

Así, tal y como se observa en nuestro estudio, se pone de manifiesto que los nińos y nińas normopeso tuvieron una EMG significativamente superior a sus compañeros con sobrepeso/obesidad respectivamente. Al analizar los datos de los niños normopeso versus sobrepeso/obesidad, estos fueron significativamente mejores que sus compañeros en cuatro estructuras: equilibrio, esquema corporal, organización espacial y organización temporal.

El grupo de niños con sobrepeso/obesidad presentaron un perfil motor por debajo de la media en las variables organización espacial y organización temporal. Así mismo se diferencian de manera significativa de los nińos de peso normal en esas mismas variables y en esquema corporal.

Se ha podido comprobar en este estudio que según avanza la edad se produce un aumento del IMC, talla y peso; esta observación está de acuerdo con la literatura (Malina et al., 2004) que parte de la idea de que el crecimiento conlleva modificaciones estructurales en todos los órganos y tejidos del organismo, reflejándose directamente en el aumento de la masa corporal. En este sentido, cabe resaltar que la masa corporal también se puede modificar en función de la dieta alimenticia y del tipo de actividad física del individuo, demostrando su sensibilidad a factores extrínsecos (Malina et al., 2004).

En las niñas, normopeso versus sobrepeso/obesidad, se encontraron diferencias significativas en cinco estructuras, motricidad gruesa, equilibrio, esquema corporal, organización espacial y organización temporal. Observándose en las niñas con sobrepeso/obesidad un perfil motor por debajo de lo normal en las variables organización espacial y organiza- 
ción temporal. La falta de una buena coordinación motriz está asociada a la falta de actividad física, característica de los niños con sobrepeso/obesidad (Tucker, 2008). Una investigación realizada por Catenassi et al., (2007) reveló diferencias significativas en algunos elementos de la capacidad coordinativa, evidenciando que los grupos de bajo peso y normopeso presentaron un desempeño mejor que los estudiantes con sobrepeso. Ese retraso en el desarrollo motor puede ocurrir como consecuencia de la reducida actividad motora y la falta de interés por el ejercicio físico, característica frecuentemente encontrada en los niños portadores de sobrepeso/obesidad.

Para Malina y Bouchard (2002), de los seis hasta los trece años de edad, la fuerza siempre debe aumentar linealmente para los niños, donde a partir de los catorce ańos ocurre una aceleración en los valores medios de corriente del período de estirón del crecimiento. Según Malina et al., (2000), GarciaMartos et al., (2004) la práctica de ejercicio con características anaeróbicas, tales como correr y actividades que envuelvan fuerza muscular, son fundamentales para el crecimiento y desarrollo motor de los niños, por lo que deben ser estimulados, ayudando a combatir el exceso de peso corporal que puede aparecer en estas edades.

Los resultados encontrados en este estudio en cuanto al desempeño motor de niños con sobrepeso/obesidad refuerzan la importancia de las actividades físico deportivas en la infancia, pues las fuerzas mecánicas gravitacionales (impacto) y las contracciones musculares inherentes a la actividad físico deportiva contribuyen a un desarrollo saludable del sistema esquelético, proporcionando un mejor control del peso corporal, sin influenciar en el crecimiento longitudinal (Ortega et al., 2008). En el presente trabajo se demostró que los niños con parámetros de obesidad y sobrepeso presentan dificultades al ejecutar la mayoría de las actividades motoras, y los efectos más visibles se producen en las estructuras donde los nińos tuvieron que realizar movimientos amplios que exigían una coordinación total del cuerpo, siendo las variables estructurales más afectadas: motricidad gruesa, equilibrio, esquema corporal, organización espacial y organización temporal. Los niños con normopeso fueron mejores que los con sobrepeso/ obesidad en todas las estructuras analizadas.

Cabe destacar que el 26,76\% de los escolares de nuestro estudio fueron clasificados como individuos con sobrepeso/ obesidad, lo que es preocupante si considerarnos las edades estudiadas. Nuestra investigación ha demostrado la necesidad de centrar esfuerzos especialmente en los nińos y nińas con sobrepeso/obesidad, así como en mejorar y ampliar la oferta de actividad física dentro y fuera del colegio, dentro y fuera del colegio. Consideramos que quizá sea necesario aumentar el número de horas de educación física escolar, con el objeto de incrementar el nivel de actividad física que garantizara un buen desarrollo de sus capacidades motoras y un mayor gasto energético, lo que redundaría, sin duda, en una mejora de su salud.

\section{Conclusiones}

A través de los resultados obtenidos, se puede concluir que los niños y niñas normopeso han demostrado un buen desarrollo motor.

Las diferencias entre los sexos en el desarrollo motor fueron significativas, contrariamente a la mayoría de los estudios encontrados sobre el tema, pudiendo haber influenciado en los resultados la elección de la prueba utilizada. Las nińas presentaron niveles de desempeño inferiores a los niños, tal vez debido al estilo de vida más activo de los niños, con juegos que permiten un desarrollo mayor de las habilidades motoras.

Al comparar la ejecución motora de cada variable entre los niños normopeso vs sobrepeso/obesidad se observaron diferencias significativas en las seis variables psicomotoras. Con relación a las áreas específicas de ejecución motora se identificó un retraso motor mayor en las siguientes estructuras: equilibrio, organización temporal, organización espacial y esquema corporal, factores estos que pueden ser atribuidos a la obesidad y sobrepeso.

La falta de actividades y el peso corporal explican esta baja ejecución motriz; a lo que se une la falta de motivación en estas edades, que cada vez se observa más en las clases de educación física.

\section{Referencias bibliográficas}

1. AHNERT, J. y SCHNEIDER, W. (2007). Development and stability of motor skills from preschool age to early adulthood: Findings of the Munich Longitudinal Study LOGIK. Zeitschrift fur Entwicklungspsychologie und Padagogische Psychologie, 39, 12-24.

2. AZNAR, S., NAYlOR, P. J., SILVA, P., PÉREZ, M., ANGUlO, T., LAGUNA, M., LARA, M. T y LÓPEZ-CHICHARRO, J. (2010). Patterns of physical activity in Spanish children: a descriptive pilot study. Child: Care, Health and Development. 37(3), 309-458.

3. BARNETT, L. M., BEURDEN, E. V., MORGAN, P. J., BROOKS, L. O, y BEARD, J. R. (2009). Childhood motor skill proficiency as a predictor of adolescent physical activity. Journal of Adolescent Health, 44, 252-259.
4. BERlezE, A., HAEFFnER, L. S. y VAlENTINI, N. C. (2007). Desempenho motor de crianças obesas; uma investiação do prcosesso e produto de habilidades motoras fundamentais. Revista Brasileira de Medicina do Esporte. 9(2), 134-144.

5. CATENASSI, F. Z., MARQUES, I., BASTOS, C. B., BASSO, L., RONQUE V. E. R. y GERAGE, A. M. (2007). Relação entre índice de massa corporal e habilidade motora grossa em crianças de quatro a seis anos. Revista Brasileira de Medicina do Esporte, Niterói, 13(4), 227-230.

6. CURY, R. y MAGALHÃES, L. (2006). Criação de protocolo de avaliação de equilíbrio corporal em crianças de quatro, seis e oito anos de idade: uma perspectiva funcional. Revista Brasileira Fisioterapia. 10(3), 103-111. 
7. EKELUND, U., SARDINHA, L. B., ANDERSSEN, S. A., HARRO, M., FRANKS, P. W., BRAGE, S., COOPER, A. R., ANDERSEN, L. B. y RIDDOCH, C. y FROBERG, K. (2004). Associations between objectively assessed physical activity and indicators of body fatness in 9- to 10-y-old European children: a population-based study from 4 distinct regions in Europe (the European Youth Heart Study). American Journal of Clinical Nutrition, 80(3), 584-590.

8. FAUSTINO, A. J. D., PIRES, T. R. y OLIVEIRA, V. R. (2004). Os efeitos da actividade física regular no desenvolvimento infanto-juvenil. Estudo no $1^{\circ}$ ciclo do ensino básico, $2^{\circ}$ ano de escolaridade. Revista do Departamento de Educação Física e Artística, 5, 515-524.

9. FEDER, K., MAJNEMER, A., BOURBONNAIS, D., PLATT, R., BLAYNEY, M. E. y SYNNES, A. (2005). Handwriting performance in preterm children compared with term peers at age 6 to 7 years. Developmental Medicine \& Child Neurology. 47(3), 163-170.

10. FONSECA, F. R., BELTRAME, T. S. y TKAC, C. M. (2008). Relationship between motor development level and variables of the development context in children. Journal of Physical Education. 19(2), 183-194.

11. GALlAHUE, D. L. y OZMUN, J. C. (2006). Understanding motor development: Infants, children, adolescents and adults ( $6^{\mathrm{a}}$ ed.). Boston: McGraw Hill.

12. GARCÍA-MARTOS, M., CALAHORRO, F., TORRES, G. y LARA, A. (2010). Efectos de un programa de entrenamiento mixto sobre la condición física en mujeres jóvenes con sobrepeso. Cuadernos de Psicología del Deporte, 10, 11-16.

13. HAYWOOD, K. M. y GETCHELL, N. (2004). Lifespan motor development. 3. ed. Champain, IL: HumanKinetics.

14. LUENGO, C. (2007). Actividad físico-deportiva extraescolar en alumnos de primaria. Revista Internacional de Medicina y Ciencias de la Actividad Fisica y el Deporte, 7(27), 174-184.

15. MALINA, R. M., PENA REYES, M. E., EISENMANN, J. C., HORTA, L., RODRIGUES, J. y MILLER, R. (2000). Height, mass and skeletal maturity of elite Portuguese soccer players aged 11-16 years. Journal of Sports Sciences, 18, 685-693.

16. MALINA, R. M. y BOUCHARD, C. (2002). Atividade física do atleta jovem: do crescimento a maturação. São Paulo: Roca.

17. MALINA, R. M., BOUCHARD, C. y BAR-OR, O. (2004). Growth, maturation and physical activity. Champaign: Human Kinetics.

18. MARTÍNEZ-LÓPEZ, E. J., LARA, A. J., CHACÓN, J. Y. y RODRÍGUEZ, I. (2009). Characteristics, frecuencies and type of physical exercise practiced by the adolescents. Sepecial attention to the obese pupil. Journal of Sport Health and Research, 1(2), 88-100.

19. MASSA, M., y RÉ, A. H. (2010). Características de crescimento e desenvolvimento. En L. R. SILVA (Ed.), Desempenho esportivo: Treinamento com crianças e adolescentes, (pp.71-108), $2^{\mathrm{a}}$ ed. São Paulo: Phorte.

20. MONTIL M.; BARRIOPEDRO, M.I. y OLIVAN, J. (2005) El sedentarismo en la infancia. Los niveles de actividad física en niños/as de la Comunidad Autónoma de Madrid. Apunts, Educación Física y Deportes, $82,5-11$

21. NATIONAL CENTER FOR HEALTH STATISTICS. NCHS. Growth curves for children birth-18 years (2005). Vital and health statistics. DNEW publ, (PHS).

22. NIETO, M. L., HERNÁNDEZ, M. T. y LAÍN. S. A. (2011). Patrones de actividad física en función del género y los niveles de obesidad en población infantil española. Estudio EYHS. Revista de Psicología del Deporte. 20(2), 621-636.

23. NILSSON, A., ANDERSEN, L. B., OMMUNDSEN, Y., FROBERG,
K., SARDINHA, L. B., PIEHL-AULIN, K. y EKELUND,U. (2009). Correlates of objectively assessed physical activity and sedentary time in children: across-sectional study. The European Youth Heart Study. BMC Public Health, 9, 322.

24. ORTEGA, F. B., RUIZ, J. R., CASTILlO, M. J. y SJÖSTRÖM, M. (2008). Physical fitness in childhood and adolescence: A powerful marker of health. International Journal of Obesity, 32, 1-11

25. OZDIRENC, M., OZCAN, A., AKIN, F. y GELECEK, N. (2005). Physical fitness in rural children compared with urban children in Turkey. Pediatrics International Journal Information. 47(1), 26-31.

26. PELLEGRINI, A. M., SOUZA NETO, S., BENITES, L. C., VEIGA, M. y MOTTA, A. I. (2003). O comportamento motor no processo de escolarizaçao: buscando soluçoes no contexto escolar para alfabetizaçao. En: Wilson Galhego and Alvaro Martin Guedes. Cadernos the Core of Education, (pp. 271-284), Sao Paulo, Brazilian: UNESP- PROGRAD.

27. ROGOL, A. D., ROEMMICH, J. N. y CLARK, P. A. (2002). Growth at puberty. Journal of Adolescent Health, 31, 192-200.

28. ROSA NETO, F. (1996). Valoración del desarrollo motor y su correlación con los trastornos del aprendizaje. Tesis doctoral. Facultad de MedicinaDepartamento de Fisiatría y Enfermería. Universidad de Zaragoza.

29. ROSA NETO, F., SILVIO, L. I. W., ANA, P. M. S. y KASSANDRA, N. A .(2010). Validaçáo dos testes de motricidade fina da escala de desenvolvimento motor EDM. Journal of Physical Education, 21(2), 152158.

30. RUIZ, L. M. (2004). Competencia motriz, problemas de coordinación y deporte. Revista de Educación, 335, 21-34.

31. RUIZ, L. M., MATA, E. y MORENO, J. A. (2007). Los problemas evolutivos de coordinación motriz y su tratamiento en la edad escolar: estado de la cuestión. Motricidad: European Journal of Human Movement. 18(4), 1-17.

32. SALLIS, J. F., PROCHASKA, J. J. y TAYLOR, W. C. A. (2000). Review of correlates of physical activity of children and adolescents. Medicine and Science in Sports and Exercise, 32(5), 963-975.

33. SHUMWAY-COOK A. y WOOLLACOTT MH. (2001). Normal postural control, En: Shumway-Cook A. y Woollacott M.H. (Ed.), Motor Control: Theory and Practical Application (pp. 163-191), 2a ed. Baltimore: Williams e Wilkins.

34. SIRARD, J. R. y PATE, R. R. (2001). Physical activity assessment in children and adolescents. Sports Medicine. 31(6), 439-454.

35. STODDEN, D. F., GOODWAY, J. D., LANGENDORFER, S. J., ROBERTON, M. A., RUDISILL, M. E., GARCIA, C. y GARCIA, L. E. (2008). A developmental perspective on the role of motor skill competence in physical activity: An emergent relationship. Quest, 60, 290-306.

36. TUCKER, P. (2008). The physical activity levels of preschool-aged children: A systematic review. Early Childhood Research Quarterly, 23, 547-558.

37. VAldiviA, A. V., CARTAGENA, L. C., SARRIA, N. E., TÁVARA, I. S., SEABRA, A. F. T.; SILVA, R. M. G. y MAIA, J. A. R. (2008). Coordinación motora: influencia de la edad, sexo, estatus socioeconómico y niveles de adiposidad en niños peruanos. Revista Brasileira de Cineantropometria e Desempenho Humano, Florianópolis, 10(1), 25-34.

38. WILliAMS, H. G., PFEIFFER, K. A., O’NEILL, J. R., DOWDA, M., MCIVER, K. L., BROWN, W. H. y PATE, R. R. (2008). Motor skill performance and physical activity in preschool children. Obesity, $16,1421-1426$. 